\title{
Tribological Behavior of High Fraction Carbon Steel Alloys
}

\author{
Rajesh S. Godse ${ }^{1,2} \cdot$ Shravan H. Gawande ${ }^{1}$ Appasaheb A. Keste ${ }^{1}$
}

Received: 19 September 2015/Revised: 11 January 2016/Accepted: 22 January 2016/Published online: 1 February 2016

(C) Springer International Publishing Switzerland 2016

\begin{abstract}
In this paper, coefficient of friction and wear rate of different steel materials are investigated and compared. Experiments are conducted on a pin-on-disk apparatus on different types of pin materials such as stainless steel SS304, stainless steel SS316, stainless steel SS202, and mild steel sliding against EN-31 disk. The experiments are performed on a group of specimens for duration of $10 \mathrm{~min}$ for various loads of 8,12 , and $16 \mathrm{~kg}$ in magnitude with disk speed of $380 \mathrm{rpm}$. The results show that the coefficient of friction varies with duration of rubbing and normal load at constant sliding velocity. In general, coefficient of friction increases for a certain duration of rubbing and after that it remains constant for the rest of the experimental time. The results obtained reveal that the coefficient of friction increases with the increase in normal load for all the tested materials. For the same operating condition, the magnitudes of the coefficient of friction and wear rate are different for different materials depending on normal load. The performance of steel with respect to wear is significantly governed by the chemical composition of material. Wear rate increases with the increase in normal load. Results have also shown that the wear of carbon steel is significantly reduced by adding more carbon content. Hence, it becomes imperative to analyze the wear characteristics of different types of steel materials, particularly SS304, SS316, SS202 and mild steel, sliding against EN-31 on a case-to-case basis in order to arrive at a more realistic assessment of wear performance.
\end{abstract}

Shravan H. Gawande

shgawande@gmail.com

1 Department of Mechanical Engineering, Modern Education Society's College of Engineering, S. P. Pune University, Pune 411001, India

2 Department of Mechanical Engineering, Army Institute of Technology, S. P. Pune University, Pune 411015, India
Keywords Friction - Wear · Pin-on-disk machine . Chemical composition

\section{Introduction}

Among various failure modes associated with steel components, wear present a unique challenge to the designer and developer of mechanical components. It is a general observation that wear resistance is the key factor governing the applicability of the material as per various requirements. Friction is the resistance to movement between any two surfaces in contact with each other. Friction is not so desirable in machines, as it destroys the effectiveness of the machines through wear and shortens its life.

The tribological characteristics, like the coefficient of friction and the wear resistance are no real material properties, but depend on the system in which these materials have to function. Number of design applications involves wear and tear process, when parts are subjected to relative motion. Wear is undesired removal of material due to mechanical action. Wear cannot be eliminated completely; however, it can be minimized. In most of the cases it is of primary concern to develop alloys and composites that possess low friction and low wear properties under dry sliding conditions against smooth metallic counterparts. (e.g., as gears or bearings) Chowdhury and Helali [1] experimentally investigated variation of friction coefficient with the amplitude of normal vibration when mild steel pin slides on different types of material such as glass fiber reinforced plastic, cloth reinforced ebonite, polytetrafluoroethylene (PTFE), rubber and mild steel. Friction coefficient is significantly high at no vibration condition than during vibration and concluded that the values of friction coefficient for said materials decreases with the increase of amplitude of vibration at different 
frequencies. Chowdhury and Helali [2] also investigated experimentally the variation of wear rate with the variation of frequency of vibration and relative humidity on a mild steel disk using specially designed pin-on-disk apparatus having facility of vibrating the disk at different frequencies. They reported that wear rate under no vibration condition is higher than that under vibration. The values of wear rate decrease with the increase of frequency of vibration. Friction coefficient and wear rate of different steel materials were investigated by Chowdhury and Nuruzzaman [3] using a pinon-disk apparatus on different types of disk materials such as stainless steel 314, stainless steel 202 and mild steel slide against stainless steel 314 pin at normal load 10, 15, and $20 \mathrm{~N}$, sliding velocity $1,1.5$, and $2 \mathrm{~m} / \mathrm{s}$ and relative humidity $70 \%$ and pointed that friction coefficient varies with duration of rubbing, normal load and sliding velocity and friction coefficient decreases with the increase in normal load. The wear in internal gears at different conditions was measured by Tunalioglu and Tuc [4] using Archard's wear modified equation and a MATLAB programme. There are many applications of friction force in engineering and technology such as automobile brakes, belt drive, wedge, vise/clamp, etc. Wang et al. [5] compared two kinds of metro brake shoe materials using a sub-scale frictional braking machine and found that the friction coefficient of braking interface has a close relationship with the velocity and pressure. In the rapid developing industry, steels continue to gain wide use as prospective functional and structural materials because of high strength, good corrosion, and wear resistance coupled with relatively low material cost. Carbon is the most important element profoundly affecting the mechanical properties of the steels. Increasing the carbon content of steels increases the hardness and strength. According to Gupta and Mishra [6] wear performance of steel significantly governed by the chemical composition of material and wear of carbon steel is distinctly reduced by adding more carbon. Gupta et al. [7] testified that wear resistance of $0.13 \mathrm{wt} \%$ plain carbon steel dependent on the microstructure and morphology of the phases.

In order to reduce friction and improve the life of machine components different techniques are applied such as surface coatings. Sivakumarana and Alankaram [8] made an attempt to describe an important role of heattreated manganese phosphate coating in the applications where temperature, corrosion, oxidation, and wear come into play. Huang et al. [9]. conducted tribological investigations on steel 20 with the carbon content and reported that the increased oxygen content on the material surface, by strain hardening contributes to decrease in the friction coefficient and increase in wear resistance. Wei and Wang [10] performed wear tests under dry sliding for 45 , $4 \mathrm{Cr} 5 \mathrm{MoSiV} 1$ and $3 \mathrm{Cr} 13$ steels at $25-400{ }^{\circ} \mathrm{C}$ and explored the relations between oxidative wear and $\mathrm{Cr}$ content and concluded that high-Cr steel are more tribo-oxides than the low or medium-Cr steels. In medium carbon micro alloyed bainitic and pearlitic steels under dry sliding wear condition with varying normal load and frequency, wear volume and wear rate of bainitic microstructure is less than that of the pearlitic steel [11]. Chowdhury et al. [12] reported that friction coefficient of stainless steel 304 sliding against aluminum, gun metal, copper, and brass varies with duration of rubbing, normal load and sliding velocity. Also it is observed that friction coefficient decreases with the increase in normal load, moreover wear rate increases with the increase in normal load and sliding velocity for all sliding pairs [12]. The wear rate of stainless steel 202 (SS202) sliding against mild steel increases with the increase in normal load and sliding velocity [13]. Chawla et al. [14]. studied tribological behavior of stainless steel 304 and gray cast iron in which it is seen that the wear behavior shown by gray cast was low due to the presence of graphite. In hardened and tempered steel with higher initial hardness and strength, exhibited better wear resistance at lower normal loads than the forced air cooled steel [15]. Friction coefficient and wear rate of copper are much lower than that of aluminum within the observed range of normal load and sliding velocity [16]. It is also noted that the wear behavior of metals and alloys at elevated temperature is influenced by wearing conditions [17].

From the past decade up to present time, many researchers had introduced advanced materials as a substitute to the conventionally used materials as these modified materials are excellent in wear and friction, light in weight and improved life span. For gear fiber and glass fiber mating with smooth or rough mild steel counter face, friction coefficient decreases with the increase in normal load and also found that friction coefficient increases with the increase in sliding velocity for both of the tested materials [18]. At identical operating conditions, the magnitude of friction coefficient of gear fiber and glass fiber are different and depends on normal load, sliding velocity and counter-face material [19]. Wear volume of the hybrid composite material increase with the increase of normal loads and sliding speed [20]. Wear behavior for all polymers i.e., polyamide 66 (PA 66), polyoxymethylene (POM), the coefficient of friction decreases linearly with the increase in pressure [21]. In wheel-rail contact, there is influence of environmental conditions and iron oxides on the wear performance, with high moisture and at room temperature (i.e., $20{ }^{\circ} \mathrm{C}$ and $10{ }^{\circ} \mathrm{C}$ ) oxide flakes would self-produce and protect the wheel-rail material from severe wear, as oxidative wear is the main wear mechanism [22]. Khun et al. [23] studied the mechanical and tribological properties of AISI D3 tool steel under deep cryogenic treatment (DCT) and reported that (DCT) is an effective way to improve the mechanical and tribological properties of the AISI D3 tool steel. Nay et al. [24] investigated the 
Table 1 Composition of pin material

\begin{tabular}{lllllllrl}
\hline & $\mathrm{C}(\%)$ & $\mathrm{Mn}(\%)$ & $\mathrm{Si}(\%)$ & $\mathrm{S}(\%)$ & $\mathrm{P}(\%)$ & $\mathrm{Cr}(\%)$ & $\mathrm{Ni}(\%)$ & $\mathrm{Mo}(\%)$ \\
\hline SS 304 & 0.022 & 1.31 & 0.31 & 0.025 & 0.017 & 18.24 & 8.60 & - \\
SS 316 & 0.031 & 1.71 & 0.28 & 0.014 & 0.026 & 16.88 & 10.47 & 2.27 \\
SS 202 & 0.095 & 8.73 & 0.37 & 0.029 & 0.028 & 15.51 & 1.73 & - \\
M. S. & 0.18 & 0.86 & 0.16 & 0.018 & 0.013 & - & - & - \\
\hline
\end{tabular}

tribological properties of epoxy composites reinforced with short carbon fiber (SCF) with different SCF contents and concluded that incorporation of SCFs is an effective way to improve the tribological properties of the epoxy composites.

From literature review, it is observed that coefficient of friction of metals, alloys, and composites show different behavior under different operating conditions.

\subsection{Scope of Study}

Stainless steels are most commonly used in machine tools for sliding and rolling (bearing) applications where low friction is expected. To reduce the wastage of energy in friction and enhance working life of tribo-pair provides higher returns to the firm. There are wide range of tribological applications of SS304, SS316, SS202, and mild steel. The effect of varying percentage of carbon content in the different steel materials can be verified by conducting experiment on Pin and disk machine.

\section{Experimentation and Procedure}

In order to investigate the effect of normal load at constant sliding velocity on the coefficient of friction for SS304, SS316, SS203, and mild steel against EN-31 and the effect of carbon content, the required experimental setup is developed and the experiments are performed on a pin-ondisk machine under dry sliding conditions. The friction coefficient and wear rate of different steel materials i.e., SS304, SS316, SS202, and mild steel sliding against disk made of EN-31 are to be investigated under given atmospheric conditions.

\subsection{Test Method}

Standard test method [25] is adopted for wear testing on a Pin-on-Disk Apparatus as per ASTM G99, (2010) American Society for Testing and Materials.

\subsection{Composition of Pin Material}

In order to explore the wear characteristics of different steel materials, it becomes necessary to know the chemical

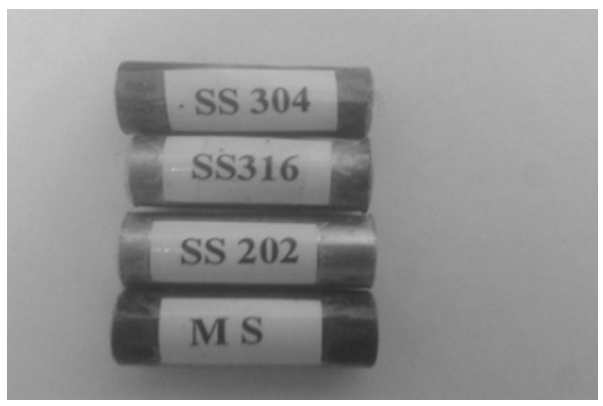

Fig. 1 Steel specimen

composition of test material. The material in the present research work is a medium carbon steel. The chemical compositions of the specimens were analyzed by an optical emission spectrometer at Metasys Testing and Calibration Laboratories LLP Bhosari, Pune, India. The chemical compositions of the materials are presented in Table 1.

\subsection{Disk}

Material used for disk is EN-31 hardened to $60 \mathrm{HRC}$ ground to 1.6 Ra surface roughness. Wear track diameter is adjustable in between 50 and $100 \mathrm{~mm}$ in steps of $1 \mathrm{~mm}$. Disk can be rotated with a speed from 200 to $2000 \mathrm{rpm}$ in steps of $1 \mathrm{rpm}$.

\subsection{Sample Preparation}

As stated above pins made of various steel materials are selected and machined with sizes $\varphi 10 \mathrm{~mm}$ and $30 \mathrm{~mm}$ long as shown in Fig. 1. The pins are held in clamping collects during conduction of test. The materials being tested fulfill all norms of G99 like dimensions, surface finish, material type, form, composition, microstructure, processing treatments, and indentation hardness.

\subsection{Load}

Experiments are carried out at normal load varying from 8, 12 , and $16 \mathrm{~kg}$ and at sliding velocity of $2 \mathrm{~m} / \mathrm{s}$. At different normal loads and constant sliding velocity, variations of friction coefficient with the duration of rubbing are to be investigated along with wear rate. 


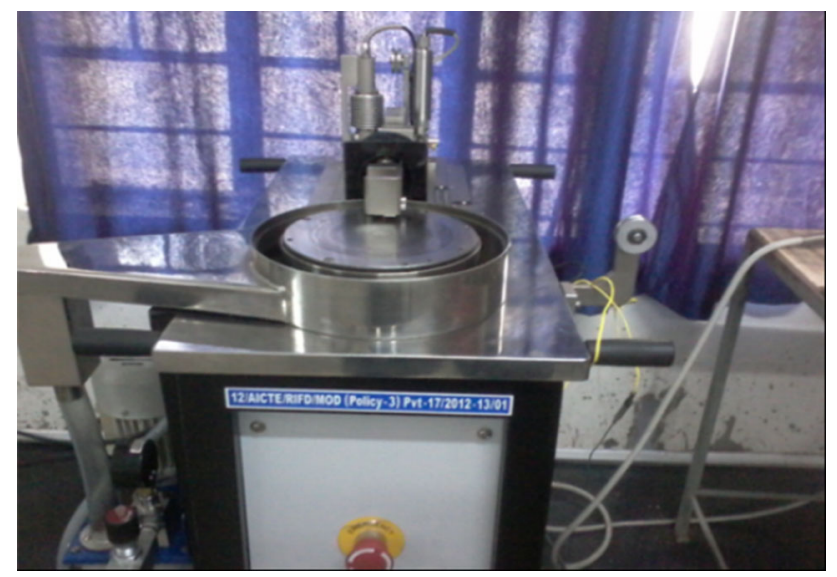

Fig. 2 Experimental set up

\subsection{Experimental Set Up}

The experimental set up comprises pin and a circular rotating disk which is placed at perpendicular with respect to the pin surface as shown in Fig. 2. The pin-on-disk machine consists of horizontal rotating disk and a calibrated dead-weight-loaded pin. The friction force and vertical displacement of pin are automatically measured in the pin-on-disk machine using a load cell and a linear variable differential transformer (LVDT). A variable speed motor rotates the disk at different speeds and is mounted in such a manner that its vibration does not affect the test. The stationary pin specimen holder is attached to a lever arm that has a pivot. Addition of weights produces a test force proportional to the mass of the weight applied. The pin specimens are cylindrical in shape as shown in Fig. 1. The pin specimen should be ground to surface roughness of $0.8 \mu \mathrm{m}$. The disk is made of hardened steel EN-31 on which the pin is held with a jaw in the apparatus and rotation is provided to disk which causes wear of the pin on a fixed path on disk. The pin is pressed against the surface of the disk with load being applied with the arm attachment provided to the apparatus. Machine is attached with a controller and data acquisition system and WINDUCOM 2010 software which give the required result.

\subsection{Wear Test Procedure}

The pin specimens are tested in Pin-on-disk apparatus as shown in Fig. 3. To perform the test specimen was clamped in jaw. Wear track diameter was fixed at $100 \mathrm{~mm}$. The rotational speed of disk was set at $380 \mathrm{rpm}$. Timer was set for ten minutes for each set of loads. Apparatus was run for $10 \mathrm{~min}$; readings were taken from the digital display. Then normal load is increased to 8,12 , and $16 \mathrm{~kg}$. Specimens from all the four materials were tested with load from

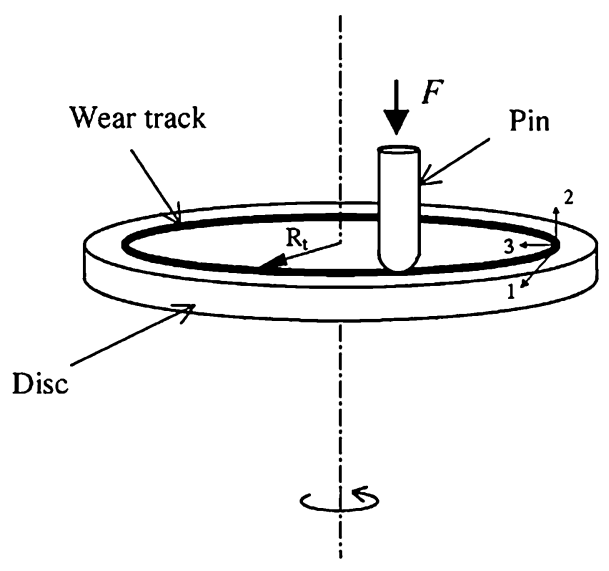

Fig. 3 Pin-on-disk wear test

8 to $16 \mathrm{~kg}$ to know the effect of load on the wear of materials. To explore the effect of carbon content on the wear specimens from materials were tested with fixed load of $16 \mathrm{~kg}$. Timer was set for $10 \mathrm{~min}$. The readings were taken and compared.

\section{Results and Discussion}

Pilot experiments are carried out using wear and friction monitor on different steel materials such as SS304, SS316, SS202, and mild steel at different loading conditions i.e., 8, 12 , and $16 \mathrm{~kg}$ at constant sliding velocity of $2 \mathrm{~m} / \mathrm{s}$. The results obtained are summarized in Table 2.

Figure 4 shows the relation between wear, coefficient of friction, temperature and frictional force against time when $8 \mathrm{~kg}$ load applied on SS304. From Fig. 4 it is observed that at the initiation of rubbing, the value of coefficient of friction is 0.2 and then it increases very steadily up to 0.443 over duration of $3 \mathrm{~min}$ of the rubbing and after that it remains constant for the rest of the experimental time. At the initial stage of rubbing, friction is low and is due to presence of a layer of foreign material on the disk surface. For $8 \mathrm{~kg}$ load applied on pin made of SS304, it is observed that, the frictional force induced is $38.2 \mathrm{~N}$ which is a nearly constant during the comprehensive experimental time. Table 3 summarizes the coefficient of friction at different loading conditions.

Figure 5 shows the comparison of the variation of coefficient of friction with the normal load. Result indicates that friction coefficient increases with the increase in normal load except SS202. For SS202 coefficient of friction decreases with increase in normal load. Increase in surface roughness and induction of large quantity of wear debris are alleged to be responsible for the increase in friction with the increase in normal load for 304, SS316, and mild steel. 
Table 2 Experimental results

\begin{tabular}{lrlll}
\hline Material & Load $(\mathrm{kg})$ & Frictional force $(\mathrm{N})$ & Wear $(\mu \mathrm{m})$ & Coefficient of friction $(\mu)$ \\
\hline SS 304 & 8 & 38.2 & 758 & 0.433 \\
& 12 & 57.9 & 1854 & 0.491 \\
& 16 & 81.2 & 1729 & 0.537 \\
SS 316 & 8 & 34.2 & 1715 & 0.427 \\
& 12 & 53.6 & 1003 & 0.457 \\
& 16 & 66.86 & 865.72 & 0.581 \\
SS 202 & 8 & 33.64 & 437.73 & 0.427 \\
& 12 & 52.17 & 1116.14 & 0.425 \\
& 16 & 69.02 & 1066 & 0.417 \\
M. S. & 8 & 26.6 & 39.42 & 0.591 \\
& 12 & 48 & -39.26 & 0.65 \\
& 16 & 52 & 265.35 & 0.72 \\
\hline
\end{tabular}
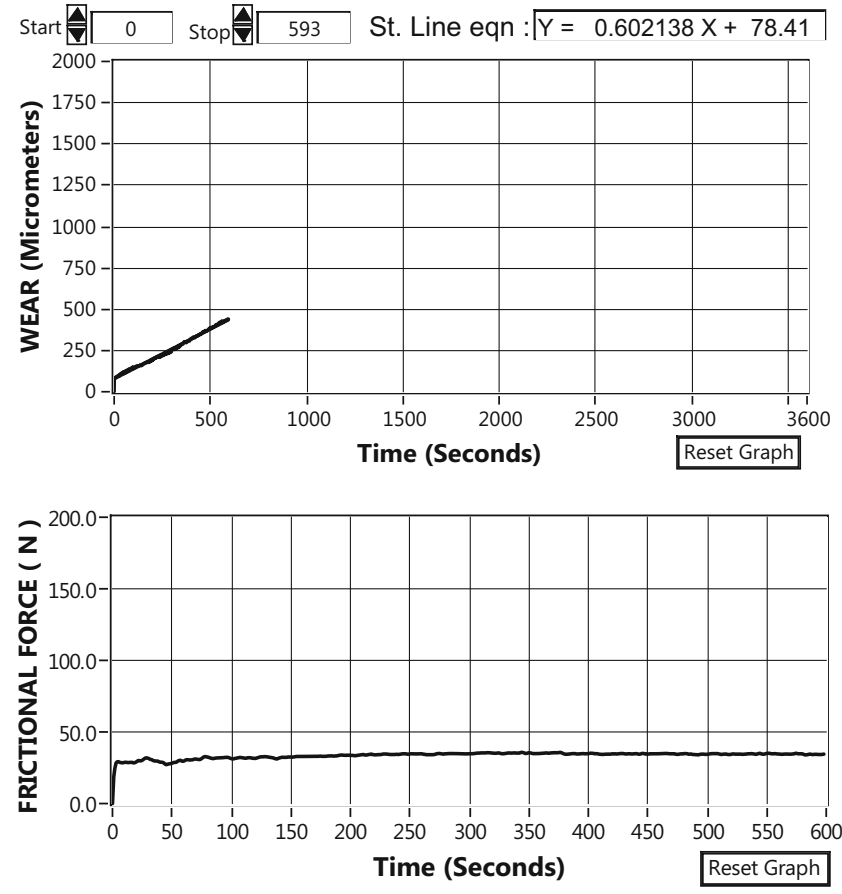

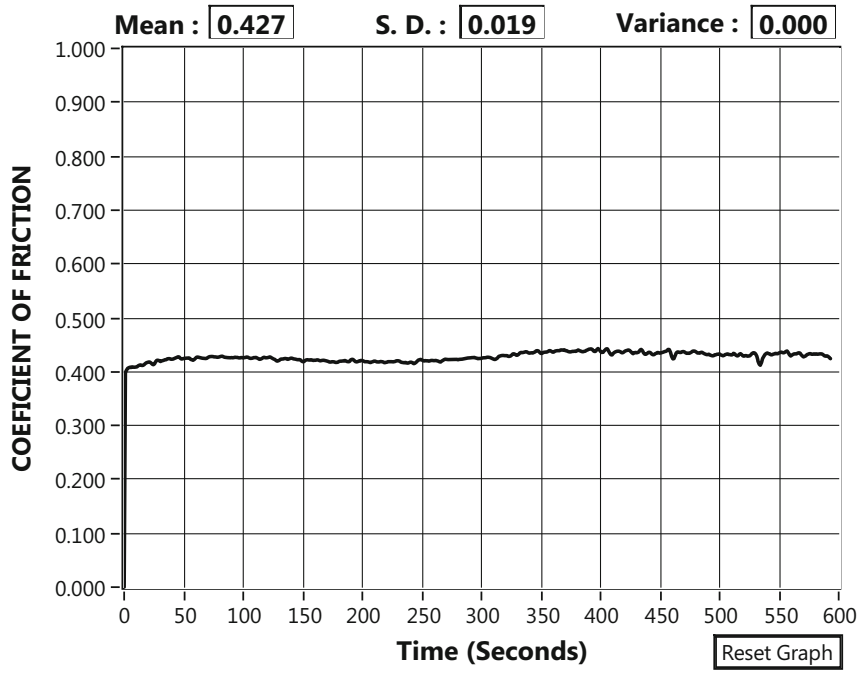

Fig. 4 Wear, coefficient of friction, frictional force of SS304 for $8 \mathrm{~kg}$ load

Figure 6 shows the variations of wear rate with the variation in normal load for SS304, SS316, SS202, and mild steel drawn as per data obtained in Table 4. Wear rate increases from 758 to 1854 micrometer and 437.73-1116.14 $\mu \mathrm{m}$ for SS304 and SS202, respectively, with the increase in normal load from 8 to $12 \mathrm{~kg}$, but further increase in load from 12 to $16 \mathrm{~kg}$ causes decrease in wear rate from 1854 to 1729 and 1003 to $865.72 \mu \mathrm{m}$. When the load on the pin is increased, the actual area of contact would increase towards the nominal contact area resulting in increased frictional force between two sliding surfaces. The increased frictional force and real surface area in contact causes higher wear. For mild steel wear decreases with increase in load from 8 to $12 \mathrm{~kg}$, and then it increases for load $16 \mathrm{~kg}$ but at very slow rate this may be due to presence of high percentage of carbon as compared to other materials. In case of SS316, it indicates that wear decreases with increase in load from 8 to $16 \mathrm{~kg}$ at very fast rate.

Table 5 indicates, wear rate in micrometer for SS304, SS316, SS202, and mild steel at different time intervals for $8 \mathrm{~kg}$ load.

From Fig. 7 it is seen that wear behavior of selected materials for $8 \mathrm{~kg}$ load at different time intervals, wear 
Table 3 Coefficient of friction at different loading conditions

\begin{tabular}{lllll}
\hline Load $(\mathrm{kg})$ & SS 304 & SS 316 & SS 202 & MS \\
\hline 8 & 0.433 & 0.427 & 0.437 & 0.591 \\
12 & 0.491 & 0.457 & 0.425 & 0.65 \\
16 & 0.537 & 0.581 & 0.417 & 0.73 \\
\hline
\end{tabular}

increases from 119.36 to $757.16,486.45$ to 1715.37 , 157.14 to 440.90 , and 23.39 to $39.42 \mu \mathrm{m}$ for $\mathrm{SS} 304$, SS316, SS202, and mild steel, respectively, with increase in time from 0 to $600 \mathrm{~s}$. For $8 \mathrm{~kg}$ load SS316 is having highest wear rate, whereas mild steel shows very slow wear rate. A similar trend of variation is also observed for SS 316 and SS 202.

Table 6 indicates, wear rate in micrometer for SS304, SS316, SS202, and mild steel at different time intervals for $12 \mathrm{~kg}$ load. Figure 8 shows wear behavior of selected materials for $12 \mathrm{~kg}$ load at different time intervals. Wear increases from 500.98 to $1853.40,227.26$ to 1003.03 ,
Table 4 Wear $(\mu \mathrm{m})$ at different loading conditions

\begin{tabular}{lcccr}
\hline Load $(\mathrm{kg})$ & SS 304 & SS 316 & SS 202 & \multicolumn{1}{c}{ MS } \\
\hline 8 & 758 & 1715 & 437.73 & 39.42 \\
12 & 1854 & 1003 & 1116.14 & -39.26 \\
16 & 1729 & 865.72 & 1066 & 265.35 \\
\hline
\end{tabular}

Table 5 Wear $(\mu \mathrm{m})$ for $8 \mathrm{~kg}$ load

\begin{tabular}{llrll}
\hline Time $(\mathrm{min})$ & SS 304 & SS 316 & SS 202 & MS \\
\hline 2 & 119.36 & 486.45 & 157.14 & 23.39 \\
4 & 284.65 & 819.82 & 211.55 & 31.08 \\
6 & 446.54 & 1492.96 & 252.99 & 34.36 \\
8 & 599.98 & 1680.41 & 365.76 & 33.2 \\
10 & 757.16 & 1715.37 & 440.9 & 39.42 \\
\hline
\end{tabular}

392.48 to 1116.14 , and 179.87 to $-34.26 \mu \mathrm{m}$ for SS304, SS316, SS202, and mild steel, respectively, with increase in time from 0 to $600 \mathrm{~s}$. For $12 \mathrm{~kg}$ load, SS304 possesses
Fig. 5 Coefficient of friction versus load

Fig. 6 Wear versus load
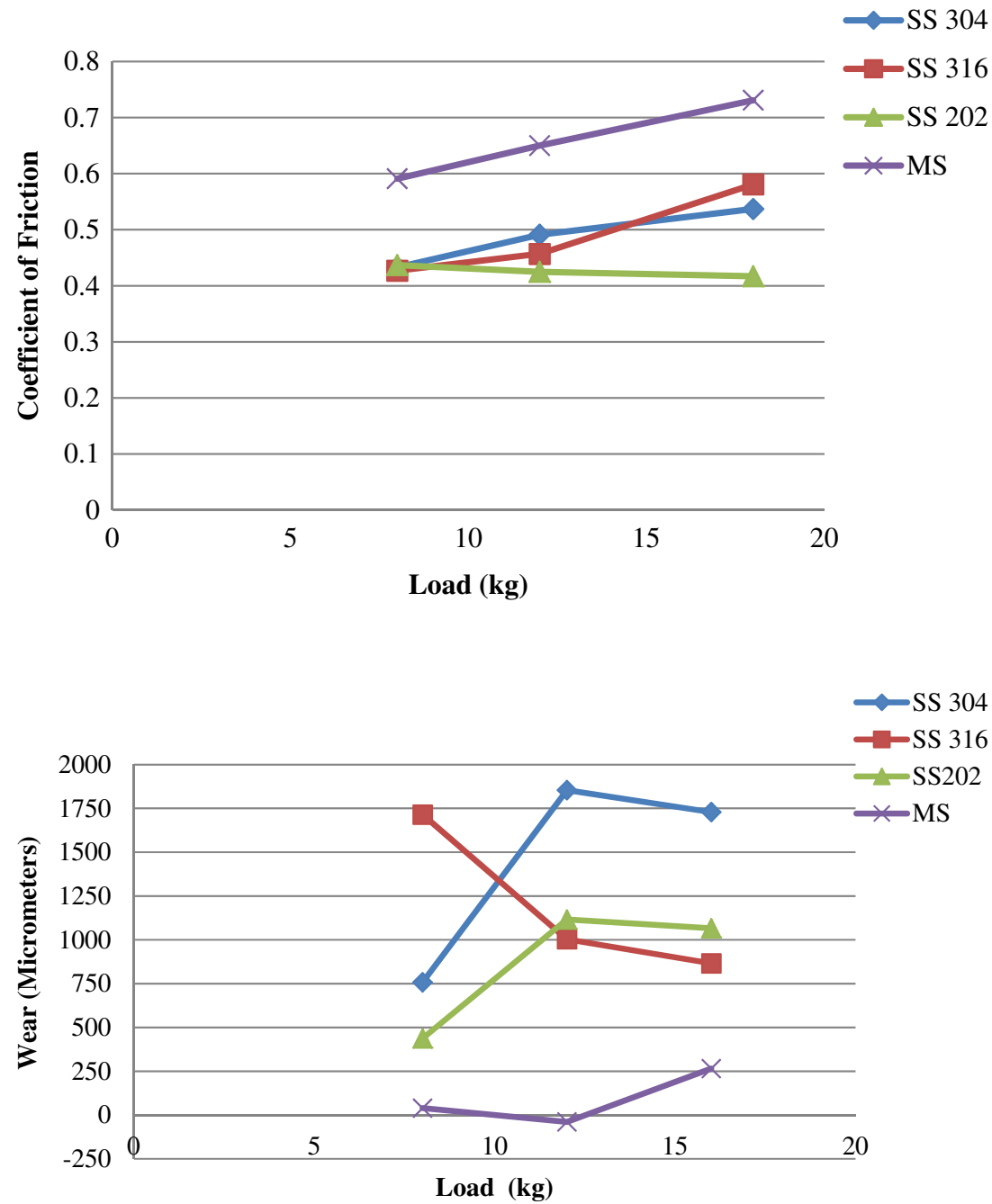
Fig. 7 Variation of wear rate with time for different materials (sliding velocity: $2 \mathrm{~m} / \mathrm{s}$, disk: EN-31 and $8 \mathrm{~kg} \mathrm{Load)}$

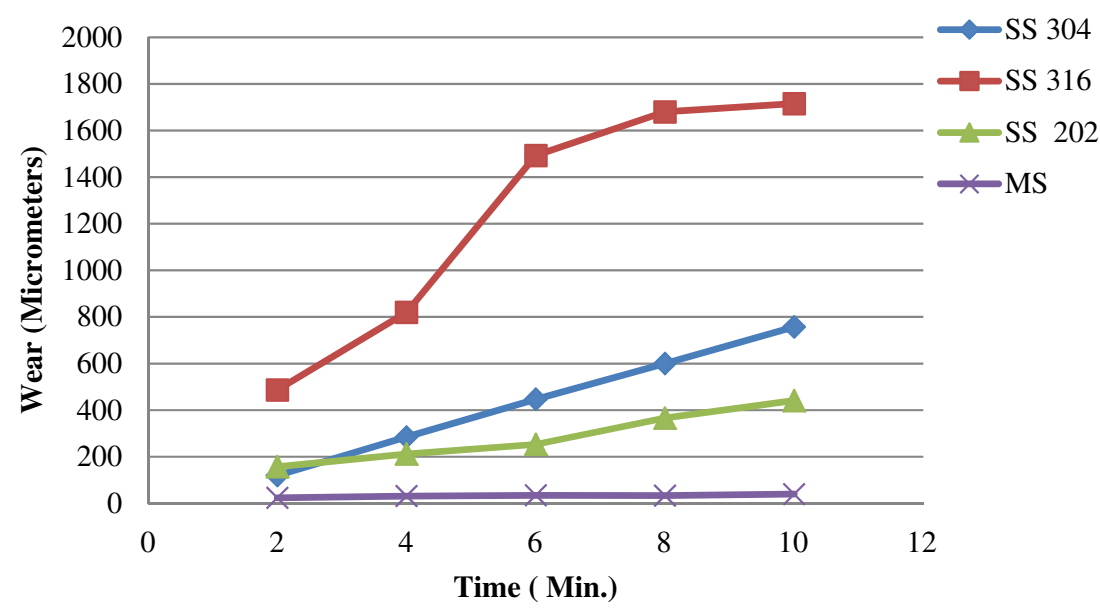

Table 6 Wear $(\mu \mathrm{m})$ for $12 \mathrm{~kg}$ load

\begin{tabular}{lrrrr}
\hline Time $(\min )$ & SS 304 & SS 316 & SS 202 & \multicolumn{1}{c}{ MS } \\
\hline 2 & 500.98 & 227.26 & 392.48 & 179.87 \\
4 & 966.94 & 476.74 & 573.54 & 173.29 \\
6 & 1346.93 & 697.63 & 759.13 & 69.34 \\
8 & 1753.85 & 852.93 & 913.94 & -46.71 \\
10 & 1853.4 & 1003.03 & 1116.14 & -34.26 \\
\hline
\end{tabular}

highest wear rate, whereas mild steel shows negative trend. For mild steel materials wear rate decreases with increase in the time.

Table 7 indicates wear rate in microns of SS304, SS316, SS202, and mild steel at different time intervals for $16 \mathrm{~kg}$ load. Figure 9 shows wear behavior of selected materials for $16 \mathrm{~kg}$ load at different time intervals. From Fig. 9 it is seen that wear increases from 815.36 to $1728.23,398.85$ to 1365.77, 449.12 to 1267 , and 208.41 to $265.35 \mu \mathrm{m}$ for SS304, SS316, SS202 and mild steel, respectively, with increase in time from 0 to $600 \mathrm{~s}$. For $16 \mathrm{~kg}$ load, SS304 possesses highest wear rate, whereas mild steel shows comparatively very slow wear rate.
Table 7 Wear $(\mu \mathrm{m})$ for $16 \mathrm{~kg}$ load

\begin{tabular}{lrrrl}
\hline Time (min) & SS 304 & SS 316 & SS 202 & MS \\
\hline 2 & 815.36 & 398.85 & 449.12 & 208.41 \\
4 & 1671.68 & 577.73 & 673.72 & 217.24 \\
6 & 1769.38 & 732.97 & 887.87 & 232.25 \\
8 & 1722.96 & 774.51 & 1066.67 & 249.35 \\
10 & 1728.23 & 1365.77 & 1267.00 & 265.35 \\
\hline
\end{tabular}

Table 8 indicates the wear induced for $16 \mathrm{~kg}$ load in different steel materials of varying percentage of carbon. Figure 10 illustrates the wear convinced for $16 \mathrm{~kg}$ load in different steel materials of varying percentage of carbon. From Fig. 10 it is observed that SS304 contains $0.022 \%$ carbon, shows highest wear when it was subjected to $16 \mathrm{~kg}$ load, whereas mild steel contains $0.18 \%$ carbon and shows minimum wear at the same loading conditions. This observation proves that the wear is strongly influenced by percentage of carbon and wear decreases with increase in percentage of carbon. If the steel possesses too little carbon percentage it means that the material has a very high load carrying capacity with higher shear strength and vice versa.
Fig. 8 Variation of wear rate with the variation of time for different materials (sliding velocity: $2 \mathrm{~m} / \mathrm{s}$, disk: EN31 and $12 \mathrm{~kg}$ load)

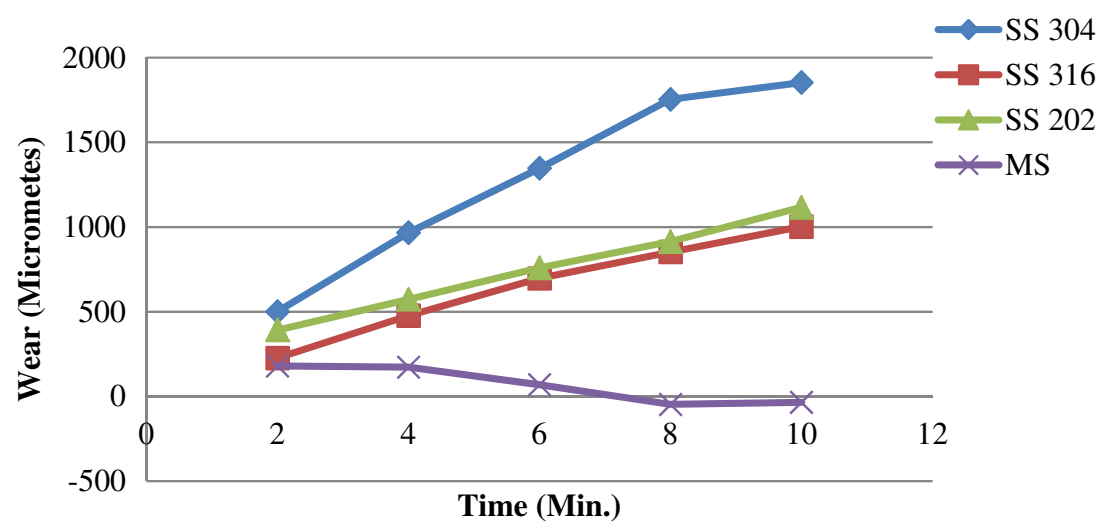


Fig. 9 Variation of wear rate with time for different materials (sliding velocity: $2 \mathrm{~m} / \mathrm{s}$, disk:

EN-31 and $16 \mathrm{~kg}$ load)

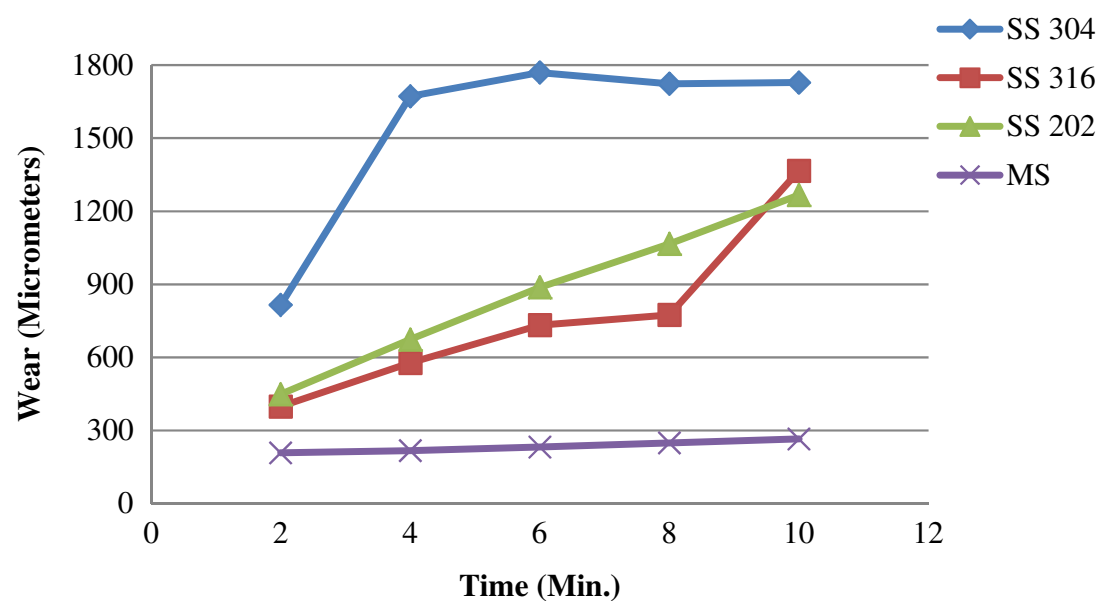

Table 8 Percentage of carbon and wear rate for $16 \mathrm{~kg}$ load

\begin{tabular}{lllll}
\hline Load & SS 304 & SS 316 & SS 202 & MS \\
\hline \% Carbon & 0.022 & 0.031 & 0.095 & 0.18 \\
Wear $(\mu \mathrm{m})$ & 1728.23 & 1365.77 & 1267 & 265.35 \\
\hline
\end{tabular}

\section{Conclusions}

In this paper, the friction and wear properties of the selected steel materials are investigated and compared. Following conclusions are drawn from the experimental investigations.

(1) Coefficient of friction increases with the increase in normal load for all tested materials except for SS202. Increase in surface roughness and large quantity of wear debris are believed to be responsible for the increase in friction with increase in normal load.

(2) The results obtained reveal that friction coefficient decreases with the increase in normal load from 8 to $16 \mathrm{~kg}$ for all the tested materials. Wear rate increases with the increase in normal load for SS304, SS316,
SS202 except mild steel. When the load on the pin increases the actual area of contact also increases towards the nominal contact area which results in increase in frictional force between two sliding surfaces. The increase in frictional force and real surface area in contact causes higher wear.

(3) At identical operating condition, the magnitudes of friction coefficient and wear rate are different for different materials depending on normal load. For 8 and $12 \mathrm{~kg}$ load SS304 shows highest wear rate whereas mild steel shows comparatively very slow wear rate. Mild steel shows negative wear trend for $12 \mathrm{~kg}$ load this may be due to entrapment of wear particles in between disk and pin. Also for $16 \mathrm{~kg}$ load, SS304 shows highest wear rate whereas mild steel shows comparatively very slow wear rate this may be due to high percentages of carbon content in it.

(4) The wear performance of steels is significantly governed by the chemical composition of material. Wear in carbon steel is significantly reduced by adding more carbon percentage.
Fig. 10 Percentage of carbon versus wear

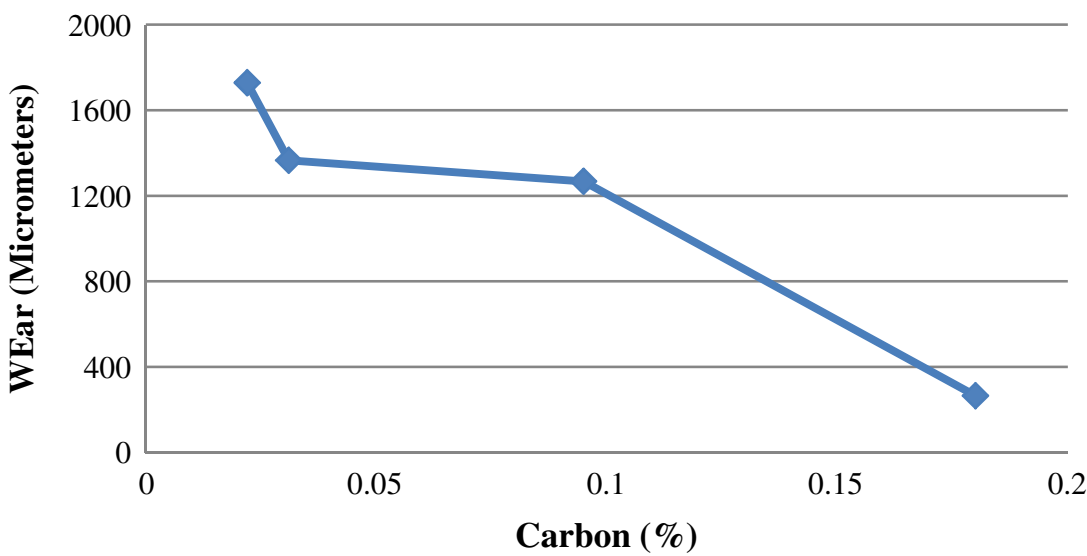




\section{Compliance with Ethical Standards}

Conflict of Interest The authors declare that there is no conflict of interests regarding the publication of this paper.

\section{References}

1. Chowdhurya MA, Helalib MM (2008) The effect of amplitude of vibration on the coefficient of friction for different materials. Tribol Int 41(4):307-314

2. Chowdhury MA, Helali MM (2007) The effect of frequency of vibration and humidity on the wear rate. Wear 262(1-2):198-203

3. Chowdhury MA, Nuruzzaman DM (2013) Experimental investigation on friction and wear properties of different steel materials. Tribol Ind 35(1):42-50

4. Tunalioglu MS, Tuç B (2014) Theoretical and experimental investigation of wear in internal gears. Wear 309(1-2):208-215

5. Wang WJ, Wang F, Gu KK, Ding HH, Wang HY, Guo J, Liu QY, Zhu MH (2015) Investigation on braking tribological properties of metro brake shoe materials. Wear 330-331:498-506

6. Gupta AK, Mishra DA (2013) An experimental investigation of the effect of carbon content on the wear behavior of plain carbon steel. Int J Sci Res 2(7):222-224

7. Gupta VK, Ray S, Pandey OP (2008) Dry sliding wear characteristics of 0.13 wt.\% carbon steel. Mater Sci Poland 26(3):617-631

8. Sivakumarana I, Alankaram V (2012) The wear characteristics of heat treated manganese phosphate coating applied to AlSi D2 Steel with oil lubricant. Tribol Ind 34(4):247-254

9. Huang SJ, Semenov VI, Shuster LS, Lin P (2011) Tribological properties of the low-carbon steels with different micro-structure processed by heat treatment and severe plastic deformation. Wear 271(5-6):705-711

10. Wei MX, Wang SQ, Chen KM, Cui XH (2011) Relations between oxidative wear and $\mathrm{Cr}$ content of steels. Wear 272(1):110-121

11. Chattopadhyay C, Sangal S, Mondal K, Garg K (2012) Improved wear resistance of medium carbon micro alloyed bainitic steels. Wear 289:168-179

12. Chowdhury MA, Nuruzzaman DM, Roy BK, Dey PK, Mostafa MG, Islam MS, Mia MR (2013) Experimental investigation on friction and wear of stainless steel 304 sliding against different pin materials. World Appl Sci J 22(12):1702-1710

13. Chowdhury MA, Nuruzzaman DM, Roy BK, Islam A, Hossain Z, Hasan MR (2013) Experimental investigation of friction coefficient and wear rate of stainless steel 202 sliding against smooth and rough stainless steel 304 couter-faces. Frict Wear Res 1(3):34-40

14. Chawla K, Saini N, Dhiman R (2013) Investigation of tribological behavior of stainless steel 304 and grey cast iron rotating against EN32 steel using pin on disc apparatus, IOSR Journal of Mechanical and Civil Engineering (IOSR-JMCE), vol. 9, no 4, pp 18-22

15. Kumara S, Bhattacharyyaa A, Mondala DK, Biswasb K, Maitya J (2011) Dry sliding wear behaviour of medium carbon steel against an alumina disk. Wear 270(5-6):413-421

16. Dewan MN, Chowdhury MA (2012) Friction coefficient and wear rate of copper and aluminum sliding against mild steel. Int Trans J Eng Manag Appl Sci Technol 4(1):29-40

17. Pauschitza A, Roy M, Franeka F (2008) Mechanisms of sliding wear of metals and alloys at elevated temperatures. Tribol Int 41(7):584-602

18. Chowdhury MA, Nuruzzaman DM, Roy BK, Samad S, Sarker R, Rezwan AHM (2013) Experimental investigation of friction coefficient and wear rate of composite materials sliding against smooth and rough mild steel counterfaces. Tribol Ind 35(4):286-296

19. Nuruzzaman DN, Chowdhury MA, Kowserb MA, Roy BK (2015) Experimental investigation on friction coefficient of composite materials sliding against SS 201 and SS 301 counterfaces. Sci Direct 255(1-6):858-864

20. Mitrovic S, Babic M, Miloradovic N, Bobic I, Stojanovic B, Dzunic D, Pantic M (2014) Wear characteristics of hybrid composites based on Za27 alloy reinforced with silicon carbide and graphite particles. Tribol Ind 36(2):204-210

21. Unal H, Sen U, Mimaroglu A (2004) Dry sliding wear characteristics of some industrial polymers against steel counterface. Tribol Int 37(9):727-732

22. Lyu Y, Zhu Y, Olofsson U (2015) Wear between wheel and rail: a pin-on-disc study of environmental conditions and iron oxides. Wear 328-329:277-285

23. Khun L, Senthilkumar D, Albert D, Lal DM (2015) Effects of deep cryogenic treatment on mechanical and tribological properties of AISI D3 tool steel. Friction 3(3):234-242

24. Nay W, Lee H, Chee Y, Xiao J (2014) Tribological properties of short carbon fibers reinforced epoxy composites. Friction 2(3):226-239

25. ASTM Standard Test Method for Wear Testing with a Pin-onDisk Apparatus, Designation: G 99-95a (Reapproved 2000) 